Historic, Archive Document

Do not assume content reflects current scientific knowledge, policies, or practices. 



\section{PLEASE OBSERVE:}

This list is intended for Nurserymen, Seedsmen, Florists and Dealers in Nursery stock only; and abrogates all previous quotations. In it we offer only such stock as we have in quantity-for other varieties see our Descriptive Catalogues. The prices are for the quantities specified; but six, fifty and five hundred of a class will be supplied at the dozen, hundred and thousand rates respectively. On bills of two hundred dollars or more in one shipment all articles will be supplied at the lowest rates quoted.

\section{OUR NURSERIES}

Cover $25^{\circ}$ acres of land, with ten large greenhouses. They are located one mile from Little Silver Depot, thirty miles from NEw YORK City, five miles from LoNG Branch and two miles from ReD BANK. To reach them from New York take the Central Railroad of New Jersey, foot of Liberty Street, or the Pennsylvania Railroad, fcot of Cortlandt or Desbrosses Streets. From Philadelphia take the Pennsylvania Railroad, Broad and Market Streets, or the Philadelphia and Reading Railroad, Bound Brook route. In all cases get tickets for Little.Silver (good on either road). Or' the Nurseries may ke approached via the New Jersey Southern Railroad, and by steamers from New York to Red Bank.

\section{THE PACKING SEASON}

Usually opens in Autumn about the first of October, and in the Spring about the first of March. All goods are carefully packed in boxes or bales, for which we make a charge sufficient to cover cost of material.

\section{OUR SHIPPING FACILITIES}

Owing to our proximity to New York City and being located upon the Pennsylvania RaILroad, the Central Railroad of New Jersey, the New Jersey Southern Railroad, with Steamboat Lines to New York City, are unsurpassed for securing quick and cheap transportation to all points North, South, East and West.

\section{WHEN NO ROUTE IS NAMED}

We forward by the one which in our judgment is the best, but purchasers will confer a favor by naming the mode of conveyance (whether by Express or Freight) and by what route they prefer to have the goods forwarded. Afer delivering to forwarders, all articles are entirely at the risk of the purchaser; and should any loss or delay occur, the forwarders alone must be held responsible. All goods are delivered at Express Office or Freight Depot at Little Silver free of cartage. Parties desiring stock shipped by express C. O. D. must remit one-quarter the amount of their purchase before shipment.

\section{CLAIMS}

If any, must be made within fifteen days after receipt of stock. Should a mistake occur, we wish to be informed of it at the earliest possible moment, that the same may be rectified without delay.

\section{TERMS CASH.}

We publish the following Catalogues:

Lovett's Guide to Horticulture, descriptive and illustrated, with colored plates, in the Spring of each year, ro cents.

Lovett's Illustrated Catalogue of Trees, Plants, Bulbs and Seeds, each Autumn, free.

Lovett's Manual of Ornamental Trees and Plants, a large and handsome book of roo pages, richly illustrated throughout; the most elaborate, accurate and complete of its kind ever issued. With beautiful colored plates, 15 cents.

Pamphlet of Pot-grown and Layer Strawberry Plants, Celery and Cabbage Plants, etc., with instructions for their cultivation, each Summer, free.

Semi-Annual Trade List, Spring and Autumn, for the Trade only, free.

\section{THE LOVETT COMPANY.}

Telegraph, Express, Money Order and Post Offices all at Little Silver, N. J.

October, 1896. 


\section{FRUIT DEPARTMENT.}

For names and descriptions of varieties see our Descriptive Catalogue. The prices of Fruit Trees are for our selection of varieties, but we will comply with the wishes of purchasers, so far as the position of our stock will permit. We cannot supply lists of selected varieties, either of Fruit or Ornamental Trees and Plants, at wholesale rates; but on such crders we will allow the Trade as great a discount on retail prices as the extent of the order will justify.

\section{PEACHES.}

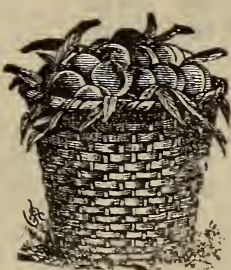

Our stock of Peach Trees is large and exceedingly fine; bright in color, smooth, straight and well furnished. Our stocks are grown from Southern natural stones exclusively, and we grow our trees upon land where Peach Trees have never before been groun. We know our trees to be absolutely free from yellows or any other disease, scale or insect. We invite special attention to our June budded trees which are large and exceedingly fine.

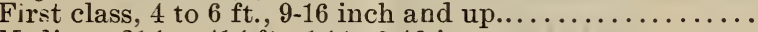

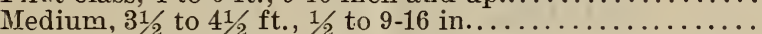

Secnnd class, $21 / 2$ to $31 / 2 \mathrm{ft}$, 3.8 to $1 / 2$ in ....................................... VARIETIES. Amsden's June (Aiexander's Early), Beers' Smock (Smock Free),
Chairs' Chnice, Champion. Crawford's Early, Crawford's Late, Crosbey, Early Rivers, Elberta, Foster, Freehold, Globe, Hale's Early, Heath Cling (Late Heath), Hill's Chili (Jenny Lind), Jacques' Rareripe, Keyport White, Large Early York (Honest John), Lemon Free, Levy's Late (Henrietta), Lovett's White, Mountain Rose, Oldmixon Free, Reed's Early Golden, Reeres' Favorite, Salway, Steadley, Stephen's Rareripe, Stump the World, Ward's Late, Wheatland.

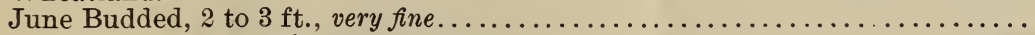

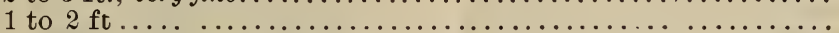

VARIETIES. Amsden's June (Aiexander's Early), Beer's Smock, Champion, Chairs' Choice, Crawford's Early, Crawford's Late, Crost,ey, Elberta, Globe, Lemon Free, Lovett's White, Mountain Rose, Oldmixon Free, Reeves' Favorite, Stump the World.

Triumph, 1st class, 4 to $6 \mathrm{ft}$

Bokara

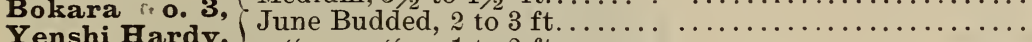

Peach Stones, Southern Natural, $\$ 1.75$ per bushel.

\section{APPLES.}

Standard, Leading Varieties, 1 st class, $3 / \pm$ in. up, 6 to $7 \mathrm{ft} . \ldots \ldots \ldots \ldots \ldots$ Medium, $5-8$ to $3 / 4$ in., 5 to $6 \mathrm{ft} \ldots \ldots \ldots \ldots \ldots \ldots$

Ruby Gem, Glowing Coal, ) 1 st class, 6 to $7 \mathrm{ft} . \ldots \ldots \ldots \ldots \ldots \ldots \ldots$ Carlough, Garfield.

$\zeta$ Medium, 5 to $6 \mathrm{tt} . \ldots \ldots \ldots \ldots \ldots \ldots \ldots \ldots \ldots$

\section{PEARS.}

STANDARD, Leading Varieties, 1 st class, $3 / 4$ in. up, 5 to $r \mathrm{ft} . \ldots \ldots \ldots \ldots \ldots$ " " "

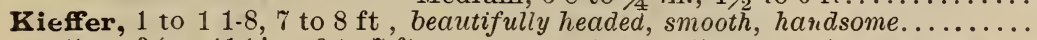

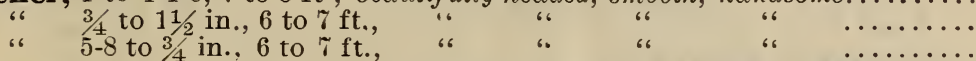

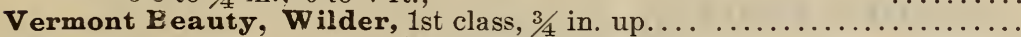
" " "

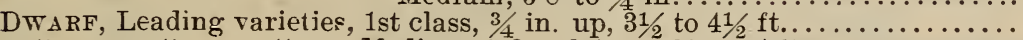
Medium, $5-8$ to $3-4$ in., $21 / 2$ to $4 \mathrm{ft} . \ldots \ldots \ldots \ldots \ldots$

\section{PLUMS.}

Staxdard VARIETIES (on Plum), 1 st class., $3 / 4$ in. up, $51 / 2$ to $7 \mathrm{ft} \ldots \ldots \ldots \ldots$

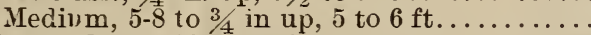
Japan Varieties (on Plum stocks), 1 st class, $3 / 4$ in. up, 6 to $7 \mathrm{ft} . \ldots \ldots \ldots \ldots \ldots$ Medium, $5-8$ to $3 / 4$ in., $41 / 2$ to $6 \mathrm{ft} . \ldots \ldots \ldots$

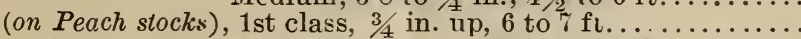

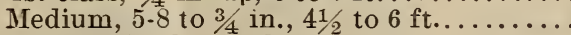
June Budded, 2 to $3 \mathrm{ft} . \ldots \ldots \ldots \ldots \ldots \ldots$ 1 to $2 \mathrm{ft} . \ldots \ldots \ldots$

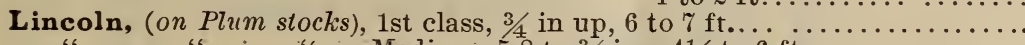
" " Medium, $5-8$ to $3 / 4$ in., $4 \frac{1}{2}$ to $6 \mathrm{ft} \ldots \ldots \ldots \ldots \ldots \ldots$ Spaulding, (on Plum stocks), 1 st class, $3 / 4$ in, up, 6 to $7 \mathrm{ft} \ldots \ldots \ldots \ldots \ldots \ldots$ Medium, $5-8$ to $3 / 4$ in., $4 \frac{1}{2}$ to $6 \mathrm{ft} \ldots \ldots \ldots \ldots \ldots$

\section{APRICOTS.}

Doz. 100 1000

$\$ 500$ 
STANDARD, Leading varieties, 1 st class, $3 / 4$ in. up...

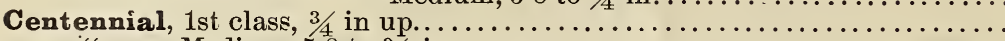
Medium, 5-8 to $3 / 4$ in

\section{QUINCES.}

Orange, 1st class, 3 to 4 ft., extra fine............................ $175 \quad 1200$

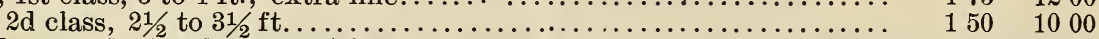

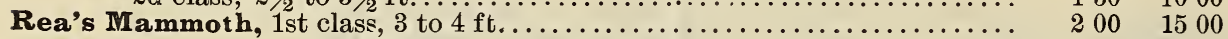

\section{HARDY ORANGE.}

Citrus trifoliata, 3 to 4 feet, well branched.

2 to 3 feet,

800

100

600

18 to 24 inches,

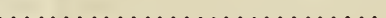

3 yrs., 12 to 18 in., branched.

$75 \quad 400$

300

3 yrs., 9 to 12 inches.

200

5000

3000

2500

NUTS.

Almond, Hardshell, 4 to $6 \mathrm{ft}$.

Russian, 3 to $4 \mathrm{ft}$.

Chestnut, American, 3 to $4 \mathrm{ft}$

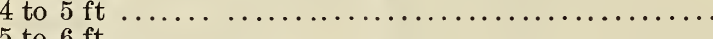

7 to $8 \mathrm{ft}$.

100

1200

600

$1 \div 5 \quad 900$

$200 \quad 1500$

$250 \quad 2000$

Japan Giant, 3 to $4 \mathrm{ft}$., trans.

350

500

Paragon, Seedlings, 3 to $4 \mathrm{ft}$

2500

Spanish, 3 to $4 \mathrm{ft}$.

750

200

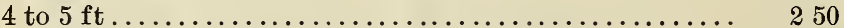

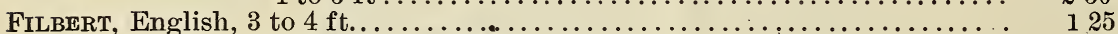

$125 \quad 800$

$150 \quad 1000$

$150 \quad 1000$

WALNUT, Black, 4 to $6 \mathrm{ft}$.

$200 \quad 1500$

6 to $8 \mathrm{ft}$.

8 to $10 \mathrm{ft}$.

Butternut, 4 to $6 \mathrm{ft}$.

$250 \quad 2000$

$175 \quad 1200$

$250 \quad 1800$

$175 \quad 1200$

English, 2 to $3 \mathrm{ft}$. .

3 to $4 \mathrm{ft}$

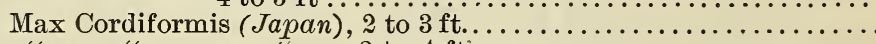

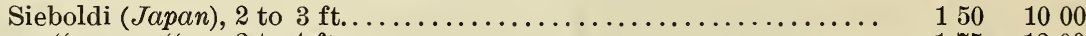

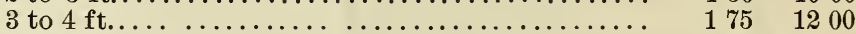

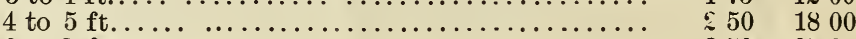

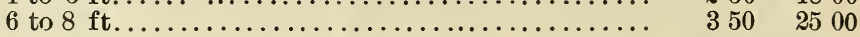

\section{GARDEN ROOTS.}

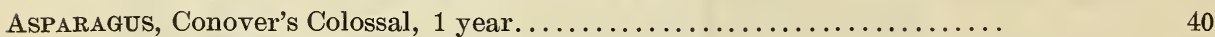

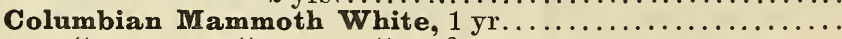

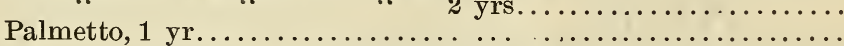

Horse Radish, sets.........

\section{RASPBERRIES.}

$100 \quad 1000$

All Scmmer (Belle de Fontenay).... \$3 50 $\$ 2500$

BRANDYwINE.................................. 100 r 00

Columbian................ 400

ConRath......................... 150

CuthBerT..................... 100

Golden QueEn................ 125

Golden Mayberry, field grown... 800

Kansas ....................... 100

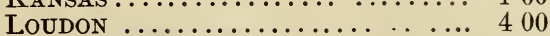

" transplanted................... 600

MARLBORO.
3000

1200

700

900

7500

700

5000

700
MILLER

. $\quad$ transplanted.............. 150 “ Colored plates (for agents).. 150

100

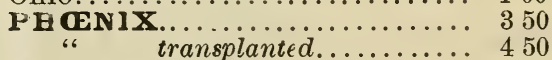

450
75

SHAFFER's COLOSSAL ............. 100

Sounegan......................... ${ }^{75} 00$

100
1000

$\$ 800$

1000

1000

600

2500

3000

500

750

500

600

600 


\section{STRAWBERRIES.}

Tied in bunches and roots straightened. No shipments until after Oct. 15th.

\begin{tabular}{|c|c|c|c|c|c|}
\hline & 100 & 1000 & & 0 & \\
\hline Inna & $\$ 150$ & $\$ 1000$ & IOWA BEAUTY & & \\
\hline$\ldots \ldots \ldots \ldots \ldots \ldots$ & 50 & 350 & Jersey Market & 400 & \\
\hline$\ldots \ldots \ldots \ldots \ldots \ldots$. & 150 & 1000 & JUCUNDA IMPROVED............ & & \\
\hline 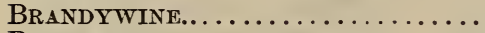 & 1 & & 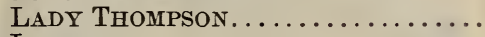 & S & \\
\hline$\ldots \ldots \ldots \ldots \ldots \ldots$ & 100 & & 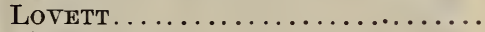 & & \\
\hline - & 50 & & SHALI & 100 & \\
\hline 然 & 5 & & RLY. & 50 & \\
\hline$\ldots \ldots \ldots$ & 50 & & aiga & & \\
\hline$\ldots \quad \ldots \ldots \ldots \ldots \ldots$ & 100 & & EXTRA EARLY........... & 3 & \\
\hline$\ldots \ldots \ldots \ldots \ldots \ldots$ & 5 & & LE. . . . . . . & 5 & \\
\hline$\ldots \ldots \ldots \ldots \ldots$ & 5 & & & 0 & \\
\hline$\ldots$ & 5 & & STER'S GEM. & 5 & \\
\hline & -5 & & ING.... . & 50 & \\
\hline & 5 & & & 1 & \\
\hline & 100 & & $\ldots$ & 50 & \\
\hline & 5 & & & 150 & \\
\hline DNER. & 5 & & $\mathrm{~T}$ & 50 & \\
\hline T & 5 & & $D^{2}$ & 50 & \\
\hline VERL & 5 & & HITE Novelty & 60 & \\
\hline ENRY V & 50 & & $\mathbf{C}_{\mathrm{S}}$ & & \\
\hline
\end{tabular}

\section{STRAWBERRY-RASPBERRY.}

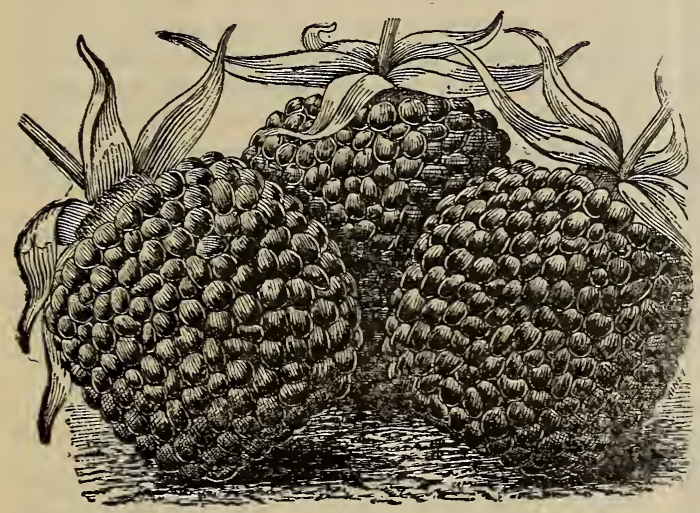

The most unique and at the same time the most beautiful berry of any kind that has as yet appeared. It comes to us from Japan, and is said to be a hybrid between the Strawberry and Raspberry. Whether this statement is correct or not we are unable to say, but that the plant is intirely hardy without protection and a prolific yielder, we can bear testimony from personal observation of plants in our trial grounds. In habit of growth the plant is distinct from both the fruits named. The root is perennial, throwing up numerous strong branching shoots, which produce large, beautiful berries from early in July until freezing weather. The foliage is composed of compound leaves formed of five or seven leaflets that have a ridged or uneven surface, light green in color, bright, clean, cheerful and pleasing, and exempt from attacks of all insects and diseases. The berries are globular, slightly oblong in form, ranging in size from $1 \frac{1}{4}$ to $1 \frac{1}{2}$ inches long and one to $1 \frac{1 / 4}{4}$ inches in diameter. The blossoms resemble in appearance small single roses, snow white in color and are as richly and deliciously fragrant as Gardenias.

Strong Pot-grown plants, doz., \$1.00; 100, \$5.00; 1000 , \$45.00.

Field grown plants, $\quad$. $\quad .60$; $\quad 3.50$; $\quad \mathbf{2 5 . 0 0}$.

Colored plates, for Agents' use, very fine. 100, $\$ 1.50 ; 1000, \$ 10.00$.

BLACKBERRIES.

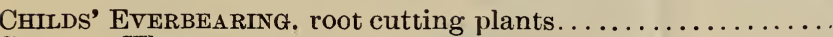

\section{CRYSTAL White,}

ELDORADO,

ERIE,

KITTATINNY,

LAWTON.

Lovett.

Maxwell,

OHMER,

RATHBUN,

SNYDER,

TAYLOR'S PROLIFIC,

WILSON's EARLY, sucker plants......

WILSON JUNIOR,

$\begin{array}{lll}66 & 66 & 66 \\ 66 & 66 & 6 \\ 66 & 66 & 66 \\ 66 & 66 & 66 \\ 66 & 66 & 66 \\ 66 & 66 & 66 \\ 66 & 66 & 66 \\ 66 & 66 & 66 \\ 66 & 66 & 66 \\ 66 & 66 & 6\end{array}$

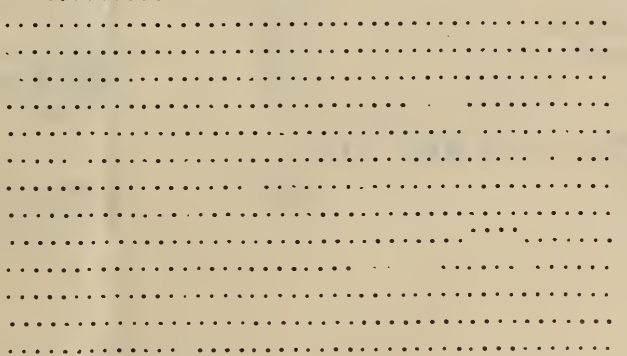

CURRANTS.

100

Black Champion, 2 yrs... . ...... $\$ 250$

BLACK NAPLES, 2 yrs ........... 210

ChERRY (Versailles), 2 yrs ......... 250

Crandall, 2 yrs................. 350

FAY's Prolific, 2 yrs............ 350
$\$ 400 \$ 3000$

150

$500 \quad 4000$

$150 \quad 1200$

$100 \quad 800$

$100 \quad 800$

$150 \quad 1200$

$150 \quad 1200$

$150 \quad 1200$

800

$100 \quad 700$

$100 \quad 800$

$75 \quad 500$

75500

LEE's ProLIfic, 2 yrs. ........... $\$ 200$

RED DUTCH, 2 yrs.

VICTORIA, 2 yrs..................... $250 \quad 2000$

White GraPE, 2 yrs............ $250 \quad 2000$ 


\section{LOGAN BERRY.}

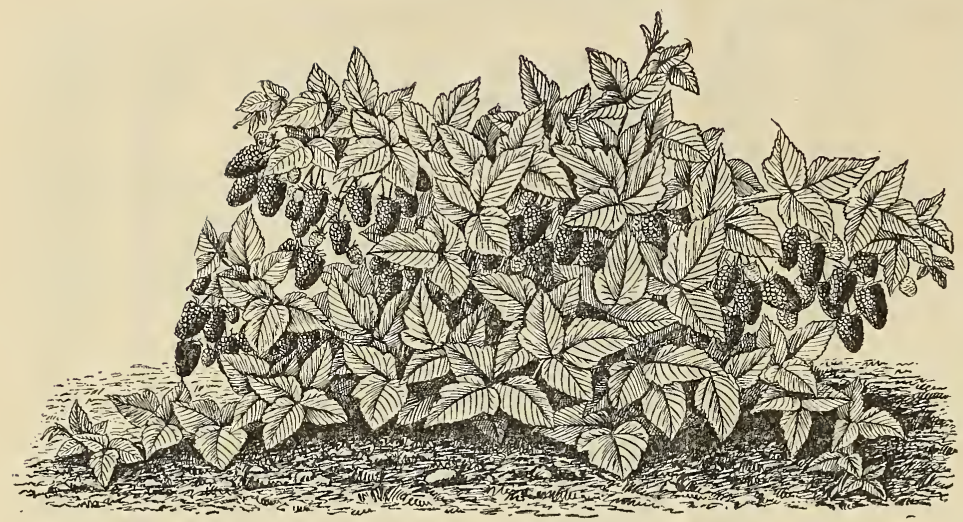

In the Logan Berry, which is a true hybrid between the Red Raspberry and Blackberry, we have a fruit destined to mark an epoch in fruit culture. Although produced in California, it has proved entirely hardy at the East and North where it has been fully tested, having been subjected to a temperature several degrees below zero, in an exposed position without the slightest protection, coming through the winters with every branch alive to the tips.

The berries are of enormous size-fully equalling the largest blackberries, which they resemble in form and structure but are deep reddish-maroon in color. In flavor, rich and sprightly-a mingling of the raspberry and blackberry, mellowed and refined, but distinct from either-melting and without core. In firmness, like a blackberry and keep a long time, either gathered or upon the bush, after ripe, before spoiling. The flavor is so luscious, novel and rich, the berry cannot fail to please everybody as a dessert fruit. For canning it is superior to all the other small fruits, and for jellies and jams it is said to be unequalled also.

The canes, which are exceedingly prolific, are of strong vigorous growth and are clothed with an abundance of large, dark green, leathery foliage and are semi-trailing in habit. (See accompanying illustration which was drawn from nature). They are without the large sharp thorns of the Blackberry but have numerous fine red spines, like those upon the Red Raspberry. What gives special value to this novel fruit is its earliness; ripening as it does at the close of the strawberry season, and in advance of all but the very earliest raspberries. The past season we gathered ripe berries of it on June 20th, in 1895 on June 24th; it continuing each year to give us ripe berries for three weeks, perfecting all its fruit, even the last, to fuli size, firmness, color and flavor.

We have now thoroughly tested this new fruit in field culture and speak from personal observation of its hardiness and other valuable properties. By reason of its great and many merits, coupled with its freedom from disease or defects, we know it to be of untold value, both for the home garden and for profit

The plants we offer were propagated from the original stock and are strictly pure and true. We caution planters against purchasing the seedling plants that are being offered by some nurseries; they are unreliable, many of them being worthless.

Good tips plants, doz., $\$ 2.00 ; 100, \$ 12.00 ; 1000, \$ 100.00$.

Colored plates, for agents' use, 100, $\$ 1.50 ; 1000, \$ 10.00$.

\section{GOOSEBERRIES.}

100

DownING, 2 yrs....................... \$4 00

Houghton, 2 yrs............... 300

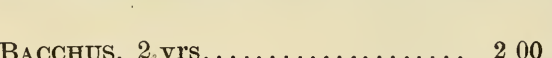

BRIGHTON, 2 yrs.................. 300

CHAMPION (Talman), 2 yrs.......... 150

ConCOkD, 2 yrs................... 150

Eaton, 2 yrs................. 500

EMPIRE STATE, 2 yrs........... 300

Green Mountain, 1 yr........... 1200

"

TREE CRANBERRY.

Bushy, 2 to $3 \mathrm{ft} \ldots \ldots \ldots \ldots \ldots \ldots \ldots$ 5 00

"6 3 to $4 \mathrm{ft} \ldots \ldots \ldots \ldots \ldots \ldots \ldots$.......... 700
1000

100

INDUSTRY, 1 vr. selected ........ \$5 00 2 ẏr.............. 600

GRAPES.

$\$ 1800$ LADY, 2 yrs................ 500

LADY WASHINGTON, 2 yrs........ 900

MOORE's EARLY, 2 yrs .......... 300

1200 Niagara, 2 yrs............... 200

Pocklington, 2 yrs.................... 200

SALEM, 2 yrs................. 250

WILDER, 2 yrs..................... 350

Woodruff, 1 yr.............. 900

WORDEN, $2 \operatorname{yrs} \ldots \ldots \ldots \ldots \ldots \ldots \ldots 200$

JUNEBERRIES.

IMPROVED DWARF, 12 to $18 \mathrm{in.....} 350$ SuCCEss, 12 to 18 in............ 500 


\section{ORNAMENTAL DEPARTMENT.}

DECIDUOUS TREES.

Doz. $\quad 100$

Acer dasycarpum. Silver-Leaved or Soft Maple, 6 to $8 \mathrm{ft}$

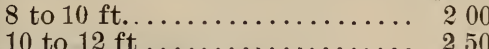

platanoides. NORWAY MaPLE, 5 to $6 \mathrm{ft} \ldots \ldots \ldots \ldots \ldots \ldots$

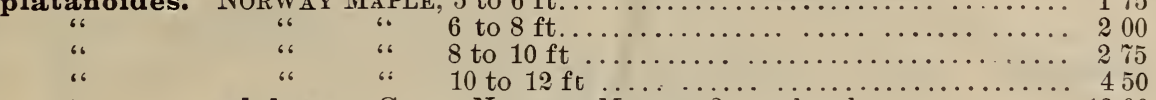

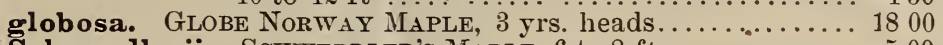

Schwerdlerii. ScHWERDLER's MAPLE, 6 to $8 \mathrm{ft} \ldots \ldots \ldots \ldots \ldots \ldots$............. 00

1500

2000

1200

1500

2500

3500

4000

6000

pseudo-platanus SYCAMORE MaPLE. 5 to $6 \mathrm{ft} \ldots \ldots \ldots \ldots \ldots \ldots \ldots \ldots \ldots \ldots \ldots \ldots .400$. 3000

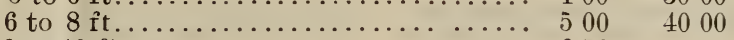

rubrum, $\mathrm{R}$.

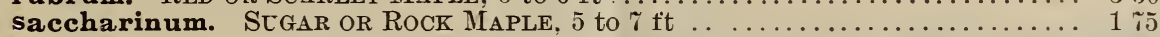

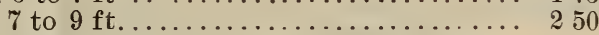

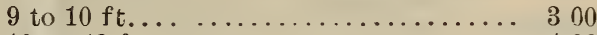

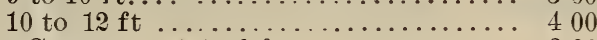

Esculus Eippocastanum. Ecropean Horse Chestrut, 5 to $6 \mathrm{ft} \ldots \ldots \ldots \ldots \ldots \ldots \ldots . . . \ldots \ldots$

6 to $8 \mathrm{ft} . \ldots \ldots \ldots \ldots \ldots \ldots+450$

8 ro $10 \mathrm{ft} . \ldots \ldots \ldots \ldots \ldots, 600$

Alnus glutinosa imperialis. IMPERIAL CUt-Leaved Alder, 8 to $10 \mathrm{ft} \ldots \ldots \ldots \ldots \ldots . . \ldots 40$

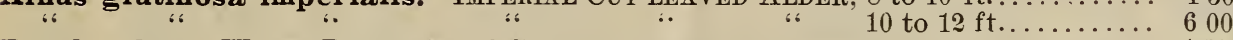

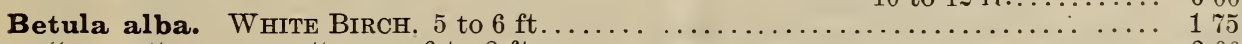

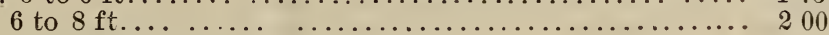

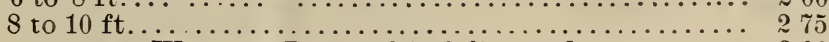

laciniata. CUT-LEAVED WEEPING BIRCH, 6 to $8 \mathrm{ft}$. stocky, ........ 300

$8 \mathrm{ton} 10 \mathrm{ft} ., \quad . . \ldots \ldots \ldots \ldots, 400$

10 to $12 \mathrm{ft}$. , " $\ldots \ldots \ldots \ldots .60060$

Catalpa speciosa. Westery Catalpa, 8 to $10 \mathrm{ft}$, stocky.......................

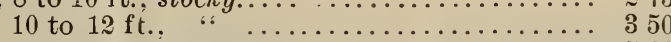

1210

1800

2200

3000

1200

1500

2000

2000

3000

4000

2000

2500

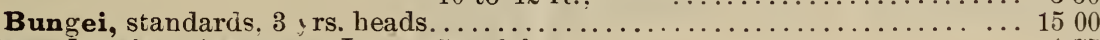

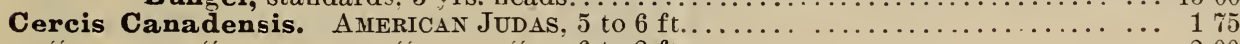

1200

1500

1000

1500

2500

3000

3500

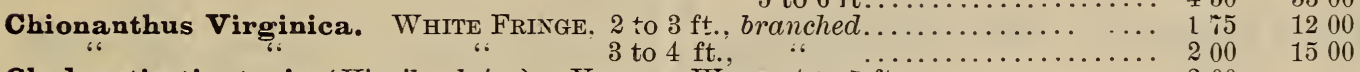

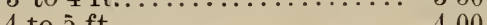

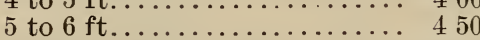

3 to $4 \mathrm{ft} ., \quad$. $\quad \ldots \ldots \ldots \ldots \ldots \ldots \ldots \ldots 200$

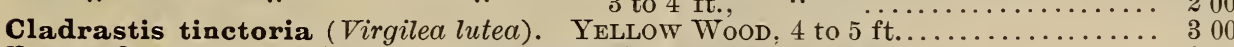

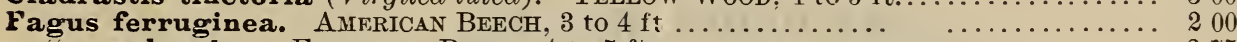

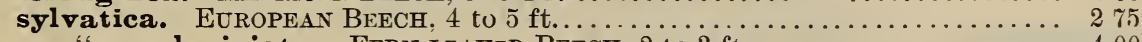

laciniata. Fern-LEA

1500

2000

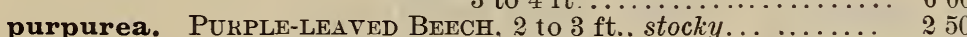

1800

2500

3000

4000

5000

2500

Riversii. RIVERs' PURPLE BEECH, 2 to 3 ft........ 350

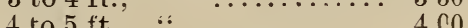

5 to $6 \mathrm{ft} ., \quad$.. $\quad \ldots \ldots \ldots \ldots \ldots \ldots$.

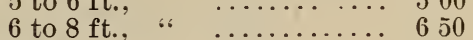

3 to $4 \mathrm{ft} \ldots \ldots .450$

tricolor. New and rery beautiful, 2 to $3 \mathrm{ft} . \ldots \ldots \ldots \ldots \ldots \ldots . \ldots 1000$

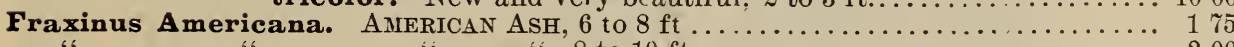

3500

1200

1500

2500

1200

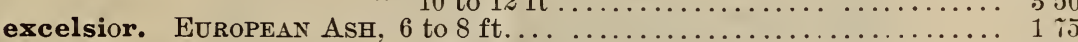

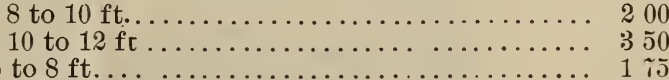

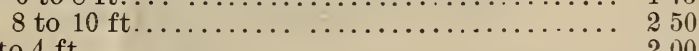

1800

1500

2000

j5 00

2000

1000

1500

2000

2500

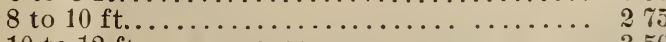

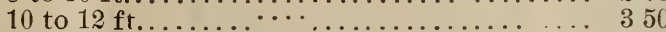

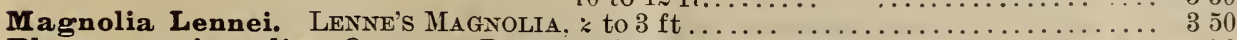

Platanus orientalis. ORIEnTaL Plane or SrCaMore, 5 to $6 \mathrm{ft} \ldots \ldots \ldots \ldots \ldots \ldots \ldots \ldots \ldots \ldots$

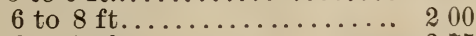


Populus canescens. Gray or Green Leaf Poplar, 10 to $12 \mathrm{ft} \ldots \ldots \ldots \ldots \ldots \ldots \ldots 275$

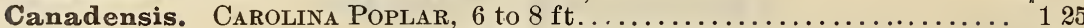

Of this popular and valuable rapid-growing shade and street

1500 tree we have a very large stock.

Van Geerti. VanGeert's Golden Poplar, 6 to $8 \mathrm{ft}$..... 150

1000 "6 8 to $10 \mathrm{ft} \ldots .200$ 10 to $12 \mathrm{ft} \ldots . .2275$

fastigiata. LOMBARDY POPLAR, 8 to $10 \mathrm{ft}$, handsome................ 150

1500

2000

1000

1500

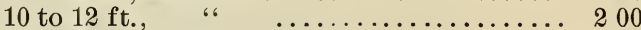

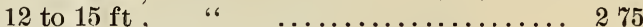

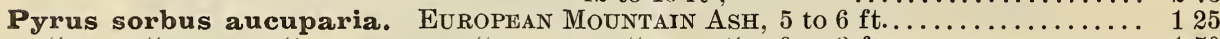

2000

750

1000

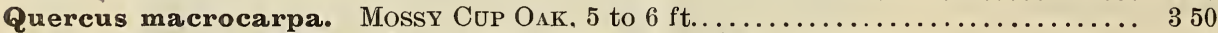

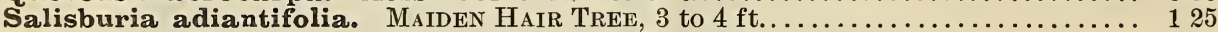

900

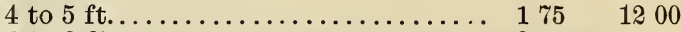

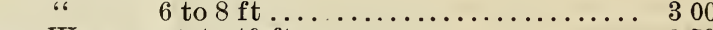

1800

1800

Salamonii. Salamon's Weeping Willow, 8 to $10 \mathrm{ft} \ldots \ldots \ldots \ldots 250$

laurifolia. LAUREL-LEAVED WILLOW, 5 to $6 \mathrm{ft}$. , bush form $\ldots \ldots \ldots \ldots \ldots \ldots \ldots \ldots$

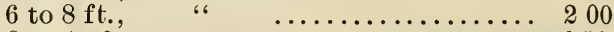

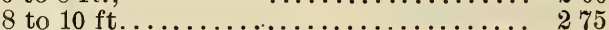

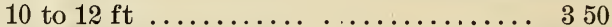

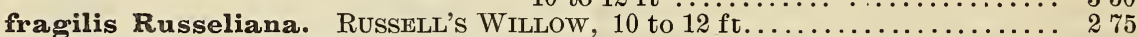

1500

2000

2500

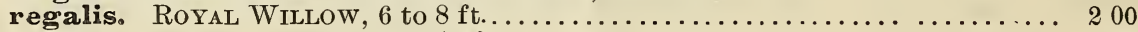

2000

1500

2000

Vernost. Russian Golden-BARKED WILLOW, 6 to $8 \mathrm{ft} \ldots \ldots \ldots \ldots \ldots \ldots \ldots \ldots \ldots .200 \quad 1500$

vitellina aurantiaca GOLDEN-BARKED WILLOW, 5 to $6 \mathrm{ft} \ldots \ldots \ldots \ldots \ldots \ldots \ldots \ldots \ldots \ldots \ldots \ldots$

1200

1800

Britzensis, ReD-BARKED WILLow, 4 to 5 to $10 \mathrm{ft} \ldots \ldots \ldots \ldots \ldots \ldots \ldots .250$

1200

1800

2500

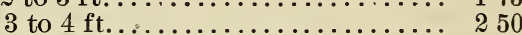

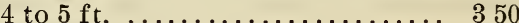

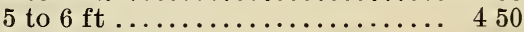

3500

5000

2500

Tilia Americana. AMERICAN LJNDEN, 9 to $10 \mathrm{ft} \ldots \ldots \ldots \ldots \ldots \ldots \ldots \ldots \ldots \ldots \ldots \ldots \ldots$

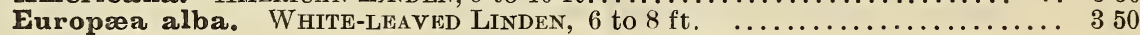

2500

3500

2000

Ulmus Americana. AMERICAN EIM, 6 to $8 \mathrm{ft}$.....

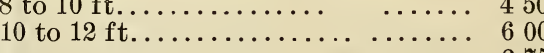

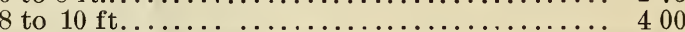

3000

\section{DECIDUOUS SHRUBS.}

Acer polymorphum. JaPANese MaPle, 3 to $4 \mathrm{ft} \ldots \ldots \ldots \ldots \ldots \ldots \ldots \ldots \ldots \ldots \ldots \ldots 250$

"

ALtheA, Double, Red, White, Purple, etc., 2 to $3 \mathrm{ft} \ldots \ldots \ldots \ldots \ldots \ldots \ldots \ldots \ldots$

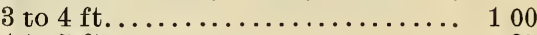

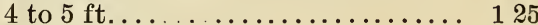

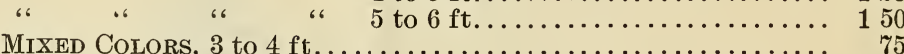

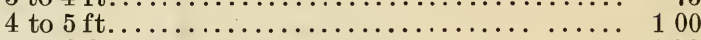

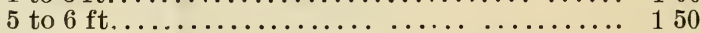

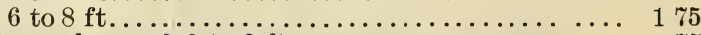

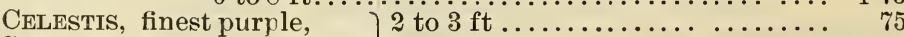

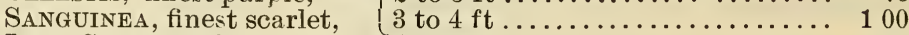

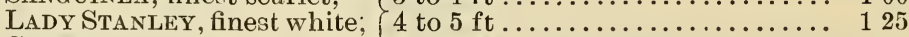

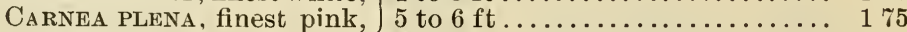

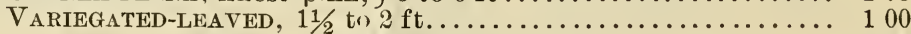

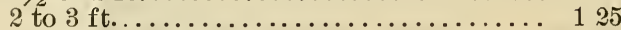

400

600

800

1000

500

700

900

1200

500

700

900

1200

600

800

Our Althceas are all grafted plants, two, three and four years old, smooth, wellbranched and handsome. The varieties are the finest in existence, selected from all that are offered in the foreign catalogues.

Andromeda mariana. StaGger Bush, $11 / 2$ to $2 \mathrm{ft}$., bushy. ................. 150

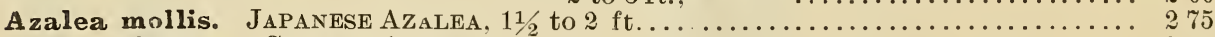

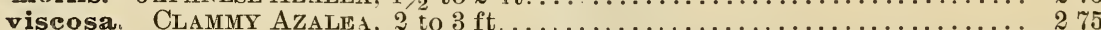

2000

2000

Berberis Thunbergii. JAPANESE BERBERRY, 6 to 9 in, trans.................. 75

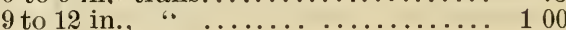

12 to 15 in.." " $\ldots \ldots \ldots \ldots \ldots \ldots \ldots \ldots$. 150

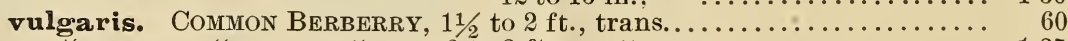


DECIDUOUS SHRUBS. Continued.

Doz.

Berberis vulgaris atropurpurea. PURPLE-LEAVED BERBERRY, 11/2 to $2 \mathrm{ft}$. trans. \$75

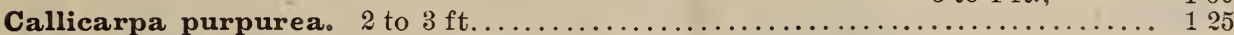

Calycanthus floridus. SwEET-SCENTED SHRUB, $11 / 2$ to $2 \mathrm{ft}$., trans.

400

2 to $3 \mathrm{ft} ., \quad$ " $\ldots \ldots \ldots \ldots \ldots \ldots 100$

3 to $4 \mathrm{ft}$., " " .............. 125

CARYOPTERIS MASTACANTHUS. BLUE SPIREA, $1 \frac{1}{2}$ to $2 \mathrm{ft}$. , bushy, field grown 1 i5

600

$A$ beautiful and valuable new shrub.

Cercis Japonica. JaPanese JUdas, 12 to 18 in., trans., bushy.............. 150

1200

1000

1200

1800

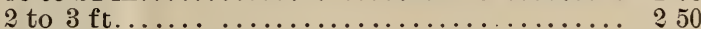

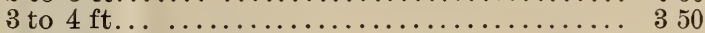

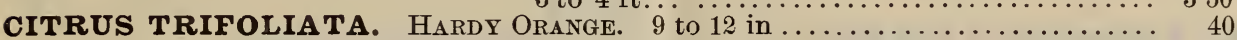

2500

200

12 to 18 in., branched..................... $50 \quad 300$

$11 / 2$ to $2 \mathrm{ft} .$. bushy................. $75 \quad 400$

2 to $3 \mathrm{ft} ., \quad$ " $\ldots \ldots \ldots \ldots \ldots \ldots \ldots, 100$

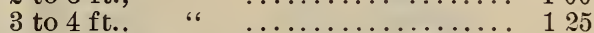

The finest hedge plant in existence.

Cornus alba (sanguinea). REv-BRANCHED DOGWOOD, 4 to $5 \mathrm{ft} \ldots \ldots \ldots \ldots \ldots \ldots \ldots$

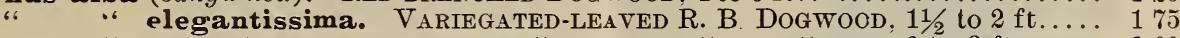

600

800

800

1200

1500

500

Cydonia Japonica. JAPANese QuINCE, 12 to 18 in., trans., bushy............... 75

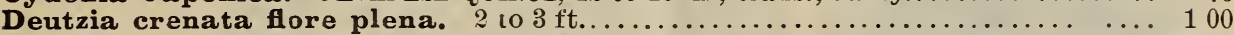

600

800

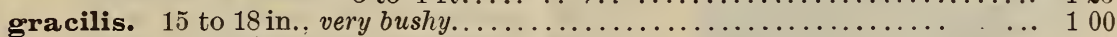

18 to 24 in., 6 ،

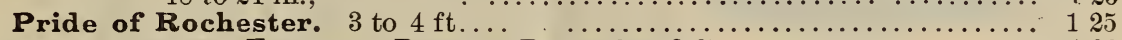

600

800

800

600

800

600

800

800

800

viridissima. GOLDEN BELL, 3 to $4 \mathrm{ft} . . .$.

Genesta Japonica. Golden FleEce, 9 to 12 in., bushy....................... 125

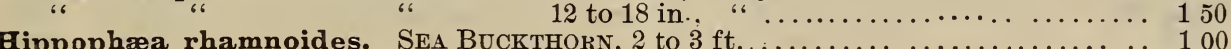

1000

600

800

HYDRANGEA PANICULATA GRANDIFLORA.

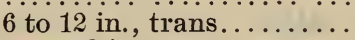

12 to 18 in., " $\ldots \ldots \ldots \ldots$. 75

18 to 24 in., "

2 to $3 \mathrm{ft} .$, "

3 to $4 \mathrm{ft} ., \quad$ " $\quad \ldots \ldots \ldots . .175$

Hуреricum aureum. Golden St. John's Wort, 18 to $24 \mathrm{in} .$, bushy........ . . . . .

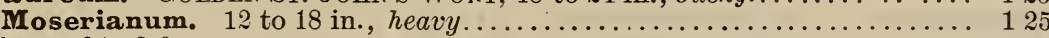

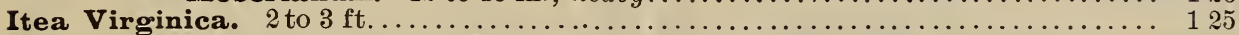

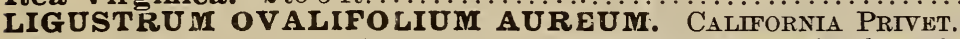

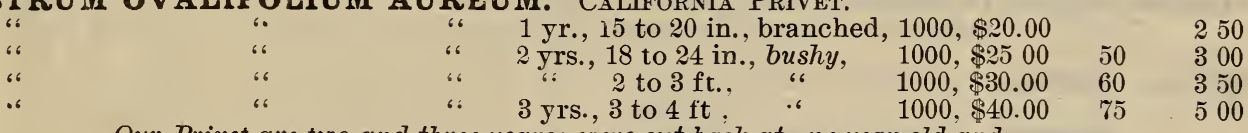

Our Privet are two and three years; were cut back at une year old and are very bushy and handsome.

Ligustrum vulgare. COMMON PRIVET, 2 to $3 \mathrm{ft} \ldots \ldots \ldots \ldots \ldots \ldots \ldots \ldots \ldots \ldots \ldots \ldots$

1000

350

500

600

800

1200

800

800

800

250

300

500

400

600

800

1000

800

1000

800

800

1000

Phlladelphus coronarius aureus. GOLDEN-LEAVED SYRINGA. 18 to 24 in............

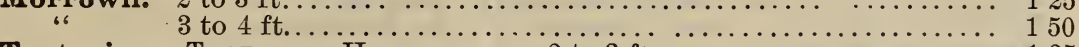

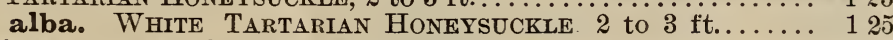

800

600

800

600

800

1000

1500

This beauciful, shrub when grown upon its own roots is vastly superior to

plants of it grown by budding; either upon peach or plum stocks.
RHUS COTINUS. PURPLE FrINGE or PURPLE MIST, 2 10 $3 \mathrm{ft} \ldots \ldots \ldots \ldots \ldots \ldots \ldots$ 
DECIDUOUS SHRUBS. Continued.

Doz.

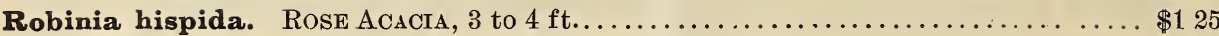

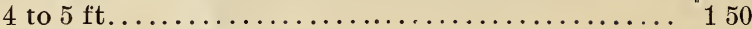

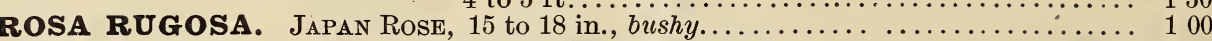

600

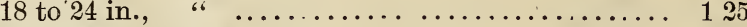

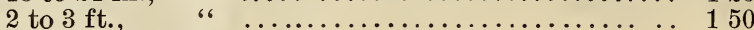

800

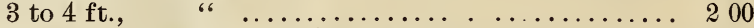

1000

1500

alba. White JaPan Rose, 15 to 18 in., bushy.................. 125

800

1000

1200

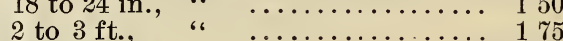

SAMBUCUS NIGRA AUREA. GOLDEN ELDER, 18 to 24 in................ 60

3511

500

800 66

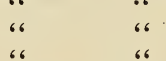

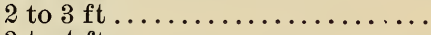

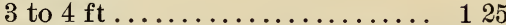

SPIREA "ANTHONY WATERER." "It is one of those exceptional novelties 1200

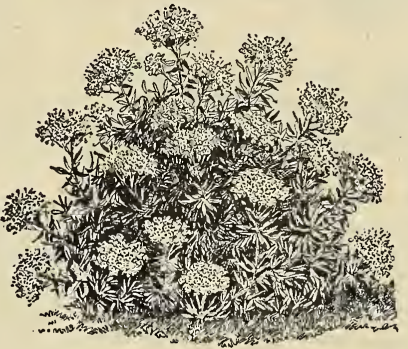

that occur only at rare intervals, adding in a conspicuous way to the rich and varied flora of our gardens. It outshines all Spiræas in brilliancy of color, a bright crimson. It is also much dwarfer and of denser growth, and a much profuser and more persistent bloomer, and is not out of bloom during summer and autumn. It originated at Knapp Hill Nursery, and Mr. Waterer quickly perceived in the variety a rare gem.

"As an instance of its continuous and abundant flowering character, I add, that on the last day of September I saw a large number in full bloom, and some plants, not more than fifteen inches high, had twenty flower clusters open at one time, and had been producing bloom in a like manner since June."-The Garden.

A varded first class certificate by the Royal Horticultural Society. and wherever exhibited.

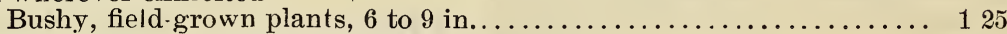

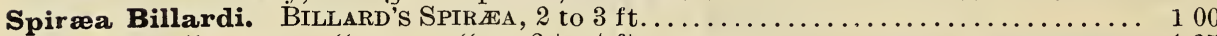

600

800

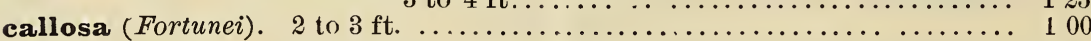

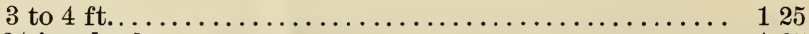

600

800

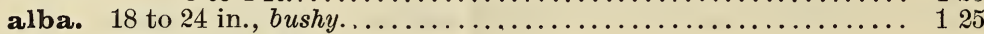

800

1000

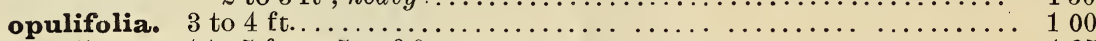

600

800

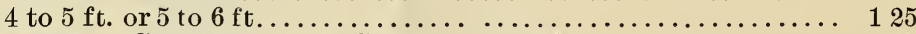

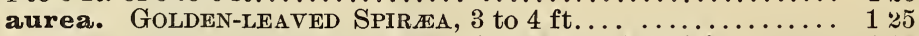

800

1200

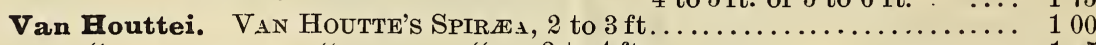

600

800

600

600

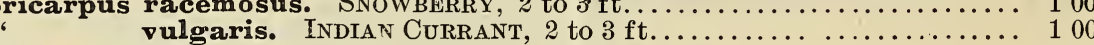

800

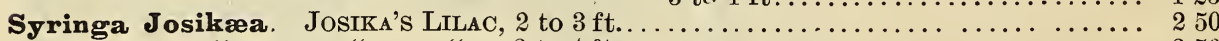

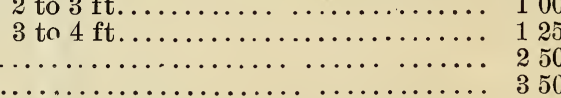

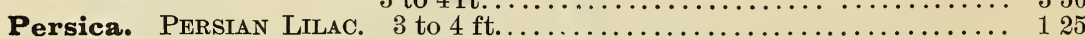

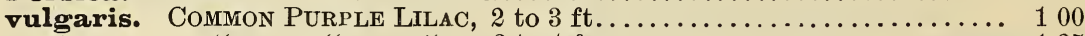

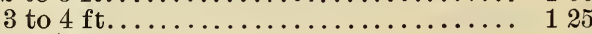

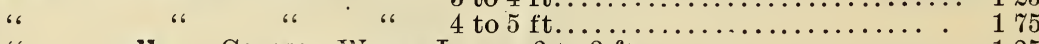

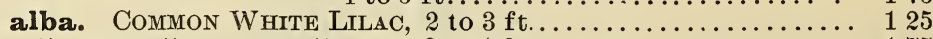

3 to $4 \mathrm{ft}$................. 175

800

600

900

1200

800

1200

Albert the Good, Charles X, Lamarck, Lemoine fi. pl., $/ 2$ to 3 ft.... 150

LUDWIG SFATH, Mad. I emoine, IM. de Dombasle, $\left\{\begin{array}{lll}2 \text { to } 3 \mathrm{ft} . \ldots . & 150 \\ 3 & \text { to } 4 \mathrm{ft} \ldots \ldots & 200\end{array}\right.$

1000

1500

500

700

600

800

1000

800

800

800

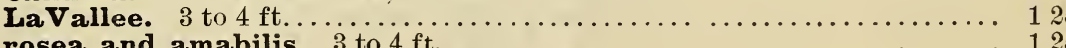

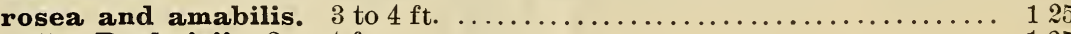

800 


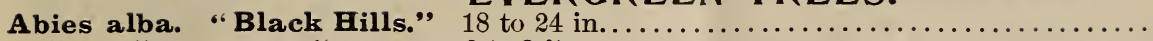

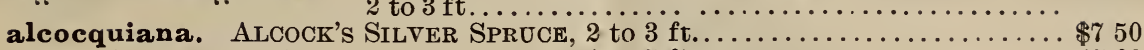

balsamea. BALSAM Frr, 2 to $3 \mathrm{ft} \ldots \ldots \ldots \ldots \ldots \ldots \ldots \ldots \ldots \ldots \ldots \ldots \ldots \ldots \ldots \ldots$

Douglasii. Douglas' Golden SPrUCE, 2 to $3 \mathrm{ft} \ldots \ldots \ldots \ldots \ldots \ldots \ldots \ldots \ldots \ldots \ldots$

EXCELSA. Norway SpruCE, 18 to 24 in., $3 \mathrm{tr}$.

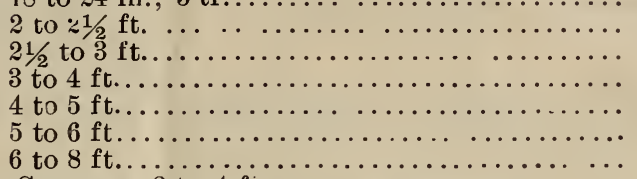

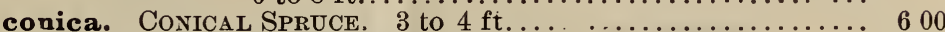

900

1200

1500

2000

2500

3500

4500

orientalis. ORIEnTAL SPRCCE, 18 to 24 in......................... 300

PUNGENS. Colorado BlUE SPRUCE, 3 vrs., 4 to 6 in..........................

Biota orientalis aurea. CHINESE GOLDEN ARBOR VIT 2,2 to $3 \mathrm{ft} \ldots \ldots \ldots \ldots \ldots \ldots \ldots \ldots$

Juniperus communis Bibernica. IRISH JUNIPER, 2 to $3 \mathrm{ft}$

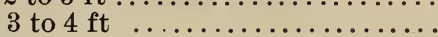

prostrata Douglasii. Douglas' GoLDEN JuniPER

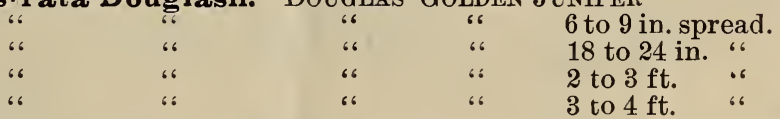

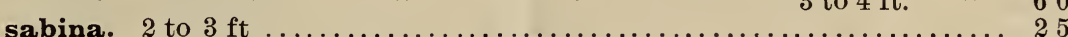

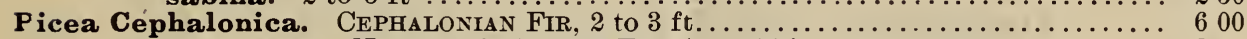

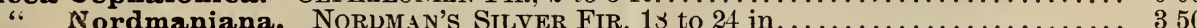

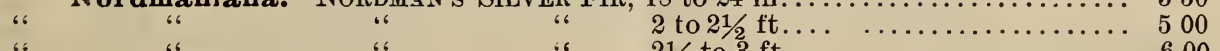

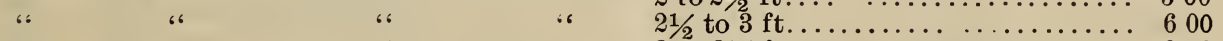

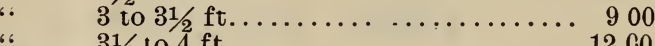

$31 / 2$ to $4 \mathrm{ft} \ldots \ldots \ldots \ldots \ldots \ldots \ldots \ldots \ldots \ldots \ldots, 1200$

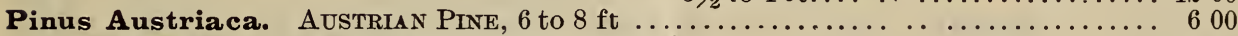

excelsa. Bhotan Weeping Pine, 3 to $4 \mathrm{ft} \ldots \ldots \ldots \ldots \ldots \ldots \ldots \ldots \ldots \ldots \ldots \ldots . \ldots \ldots$

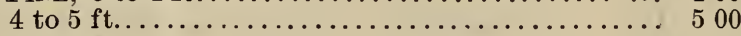

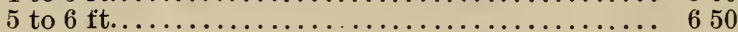

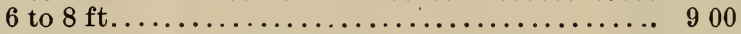

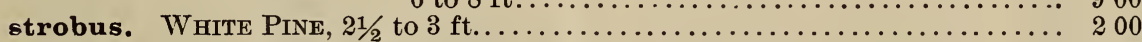

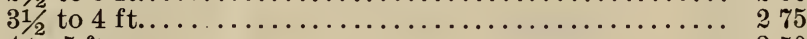

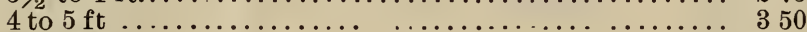

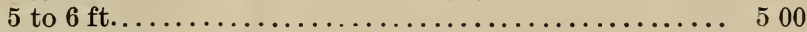

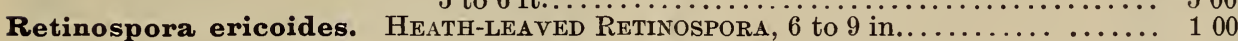

9 to 12 in............... 125

15 to 18 in., 2 tr............... 175

18 to 24 in., 2 tr.......... 250

2 to $21 / 2 \mathrm{ft}$, shorn.............. 400

600

900

1500

1000

1500

800

1800

2500

2500

1500

2000

25 00

600

800

1200

1800

filifera. WEEPING RETINOSPORA, 18 to 24 in.................... 250

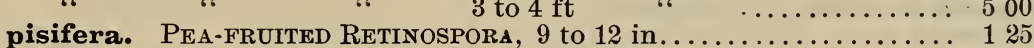

800

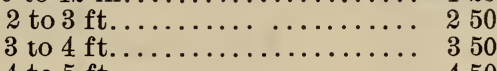

4 to $5 \mathrm{ft} . \ldots \ldots \ldots \ldots \ldots \ldots \ldots \ldots, 450$

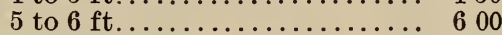

aurea. Golden PEa-fruIted RetinOSPORA, 2 to $3 \mathrm{ft} \ldots \ldots .400$

plumosa. Plume-LIKE RETINOSPORA, 9 to 12 in ................

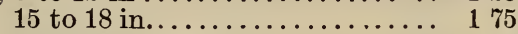

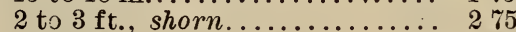

3 to $4 \mathrm{ft} .$, s6 $\ldots \ldots \ldots \ldots \ldots \ldots 450$

4 to $5 \mathrm{ft.}, \quad$ " $\ldots \ldots \ldots \ldots \ldots 600$

argentea. SILVER-TIPPED, PLUME-LIKE Ret., 6 to 9 in...... 125

aurea. GOLDEN-TIPPED

$\begin{array}{ll}66 & 66 \\ 66 & 66 \\ 66 & 66 \\ 66 & 66\end{array}$

18 to 18 in .... 250

2 to $3 \mathrm{ft}$ shorn 500

"6 6 to 9 in ...... 100

600

1000

1500

2000

2500

3500

2000

3000

4000

6000 
EVERGREEN TREES. Continued. Doz.

Taxus baccata. EngLISH YEw, 20 to 24 in., shorn...................... $\$ 500$

" "

$21 / 2$ to 3 ft., "6 $\ldots \ldots \ldots \ldots \ldots \ldots \ldots \ldots \ldots \ldots, 750$

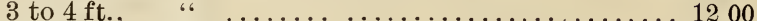

Hibernica. IRISH YEW, 12 to 18 in ............................ 400

"

Thuja occidentalis. AMERICAN ARBor Vit 1,12 to 18 in., 2 tr....................... $\$ 400$

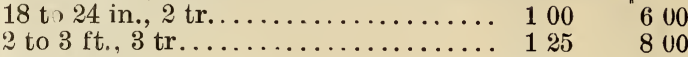

3 to $4 \mathrm{ft} \ldots \ldots \ldots \ldots$........ 450

4 to $5 \mathrm{ft} . \ldots \ldots \ldots \ldots 60$

Columbia. Sillver-TIPPED ARBor Vite, 12 to 15 in., 2 tr.... 300

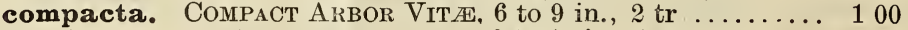

600

1000

1500

2000

12 to 15 in., $2 \operatorname{tr} \ldots \ldots \ldots \ldots 20$

20 to 24 in., 3 tr........... 300

conica densa. 6 to 9 in., 2 tr..............................

9 to 12 in., 2 tr....................... $150 \quad 1000$

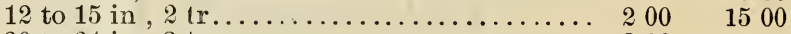

20 to 24 in.. 3 tr.................... $300 \quad 2000$

Douglasi. Douglas' Golden ARBor Vit 2,9 to 12 in., 2 tr.. $200 \quad 200$

2000

12 to 15 in., 2 tr.. $300 \quad 2000$

Elwangeriana. TOM THÜв ARBor Vite, 6 to 9 in., 2 tr.... 100

500 9 to 12 in., 2 tr... 125 18 to 24 in.. $3 \mathrm{tr} . .2200$ 2 to $3 \mathrm{ft} ., 3 \mathrm{tr} . . .3300$

800

1500

2000

globosa. Globe ARBOR Vit

2000

2000

Hoveri. Hover's GOLDEN A 20 to 24 in., 3 tr.............. 300

3000

pyramidalis. PYRAMIDAL ARBOR VIT E, 15 to 18 in., $2 \operatorname{tr} \ldots . .2150 \quad 1000$ 2 to $3 \mathrm{ft} ., 3 \mathrm{tr} . \ldots \ldots 250 \quad 1800$

Sibirica. Siberian Arbor Vit «, 18 to 24 in., 3 tr.............. $200 \quad 1500$

4 to 4 tt............. 350

2000

3000

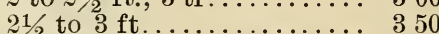

3 to $31 / \mathrm{ft} \ldots \ldots \ldots \ldots \ldots .400$

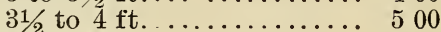

4 to $5 \mathrm{ft} . \ldots 600$

Vervæneana. Verv झENE'S ARbor Vitae, 9 to 12 in., 2 tr... 125

3500

800

\section{EVERGREEN SHRUBS.}

Azalea amœna superba grandiflor a, 15 to 18 in $\ldots \ldots \ldots \ldots \ldots \ldots \ldots \ldots \ldots \ldots$. 350

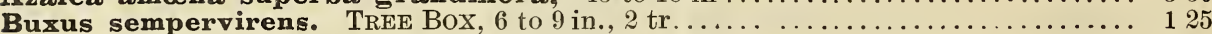

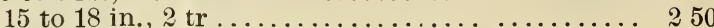

foliis aureis. NEW GOLDEN-LEAVED Box, 4 to 6 in. 2 tr... 125

800

800

9 to 12 in....... 250

". 15 to 18 in ..... 500

longifolia. Long-LEAVED TreE Box, 6 to 9 in., 2 tr....... 125

800

1200

9 to 12 in.......... 175

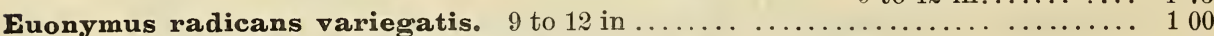

600

800

1200

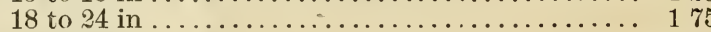

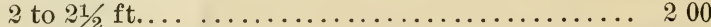

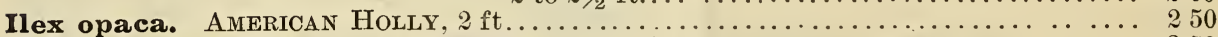

Kalmia angustifolia. SHEEP LAUREL, 2 to $21 / 2 \mathrm{tt} \ldots \ldots \ldots \ldots \ldots \ldots \ldots \ldots \ldots \ldots \ldots$

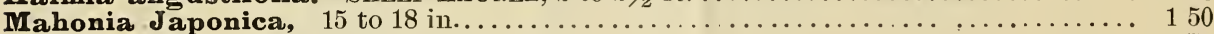

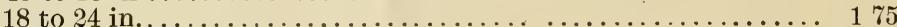

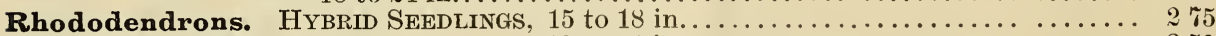

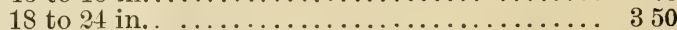

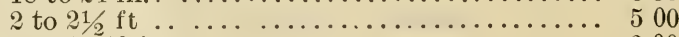

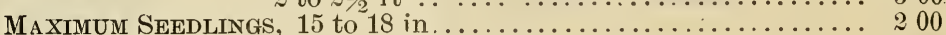

NAMED, Finest hardy varieties, 15 to $18 \mathrm{in.}$. with buds.......... 600

1500

900

1200

2000

2500

1500

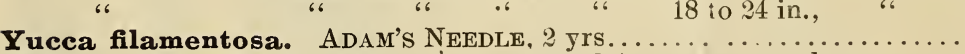




\section{VINES AND CREEPERS.}

Doz.

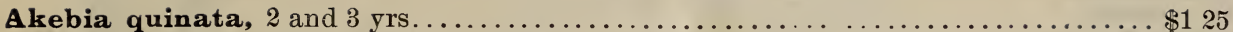
AMPELOPSIS quinquefolia. VIRGINIA CREEPER, 2 yrs $\ldots \ldots \ldots \ldots \ldots \ldots \ldots \ldots \ldots \ldots \ldots$

Grown to stakes with tops of from 3 to 5 feet.

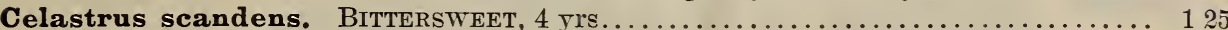

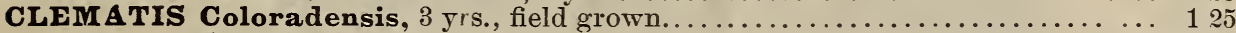

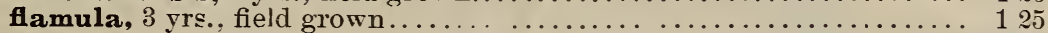

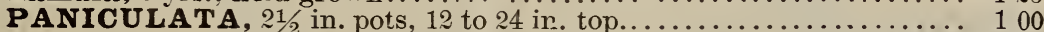

strong, field grown, 2 yrs...................

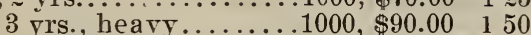

800

800

800

800

500

800

Clematis paniculatı is the most valuable addition to the list of hardy vines that has been made within the last 25 years.

Lonicera Halleana. HALL'S JAPAN HONEYSUCKLE, 2 and 3 yrs.............. 100

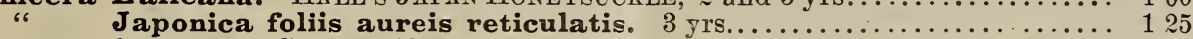

Sinensis. ChINese EvergReen HonersuckLe, 3 yrs................... 100

Lycium Sinensis. CuInese Matrimony VINE, strong, field grown, 2 to $3 \mathrm{ft} \ldots \ldots \ldots \ldots$

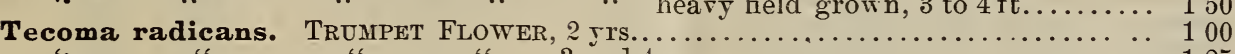

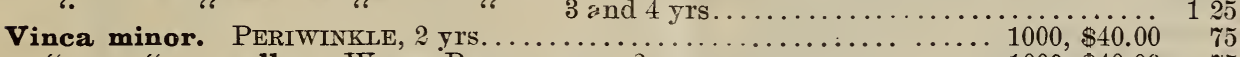

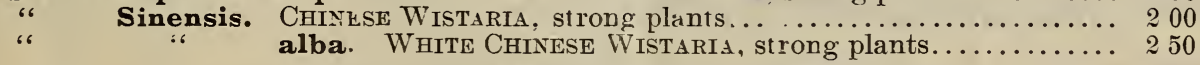

\section{HARDY HERBACEOUS PLANTS.}

Our stock of Hardy Herbaceous Plants is very large and the plants are exceedingly fine, many being grown in pots. Those in need of a large number will please send lists for estimate. We here offer but a comparatively small number of the varieties we have in stock.

Achillea Ptarmica serrata fl. pl. "66 The Pearl.", millifolium roseum.

Achorus calamus variegata. VARIEGATED SWEET FLAG.

Alysoum saxatile compactum. DENSE GOLDEN TCFT.

Alysoum saxatile compactum. DENSE GOLD

Anthemis tinctoria. GOLDEN MARG
Arenaria verna. SPRING SANDWORT.

Arenaria verma. SPRING SANDWORT.......................

Amsonia Tabermamontama, strong fleld grown plant
Aquilegia chrysantha. GOLDEN-SPCRRED COLUMBINE.

A quilegia chrysantha. GOLDEN-S

Aster Nova-A ngla rosea. NEW ENGLAND ROSE ASTER.

Istilbe Japonica. (Spirce Japonica). JAPANESE ASTILBE.

Baptisia australis.

Boceonia cordata (Japonica)

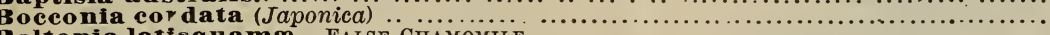

Boltonia latisquamie. FALSE CHAMOMILE.

Calamintha glabella

Callirrhoe involuerata. CRIMSON PoPPY MALLOW

centaurea montana. PERENNIAL CORNFLOWER...

Cerastium Bierbersteinii. MOUSE. EAR CHICKWEED

Chelone Lyonil. LYON'S TURTLE HEAD

Chrysauthemum lacustre....

Clematis Davidiama, 2 yrs

Coreopsis lanceolata. GOLDEN WA 3 and....

\section{“ rosea.. verticeliata.}

Delphin $\mathbf{u m}$ sinensis grandiflorum. CHINESE HYBRID LARKSPUR.

Dianthus plumarius roseus plenus. SCOTCH PINK.

" H

Digitalis purpurea. ForgLore.

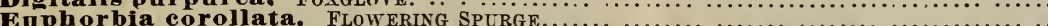
Fuphorbia corollata. FLOWERING

Helenium antummale. SNERZE WEED...

Heliantbus latiforus

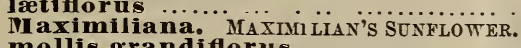

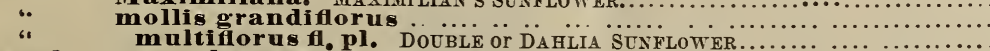

Helianthemum vulgare fl. pl. SUN ROSE.

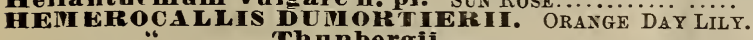

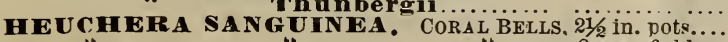




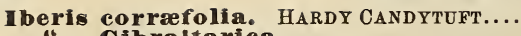
Gibraitarica. Tenoreana.... EVERGREEN CANDYTUFT

IEIS Florentina ORCHID or FRAGRANT IRIS

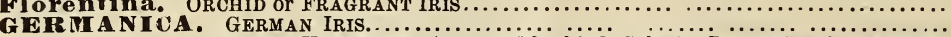

GER M A N I CA. GERMAN IRIS............. Bluebird, celeste, Donna Marie, LaTendre Mad. Cherau, Pallida speciosa, Sampson, Sappho, etc.

MIXED...

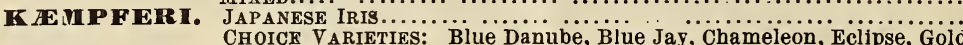
Band, Pyramid, Mahogany, Wm. Tell, etc. MIX ED..

nudicaule. DWARF IRIS.

Kosteletzk ya Virginica. PINK BEAUTY

Lathy rus Jatifolius. Everlasting PEa

Lavend u la vera. LAVENDER

Liatris pictnostachya. KANSAS GAY FLOWER

Lychnis flos-cuculi plenissima semperfiorens.

vigegria splendens f pl. RAGGe ROBIN.

Tremarda didyma coceimea.

Mountia vulgaris. PRICKLY PEAR

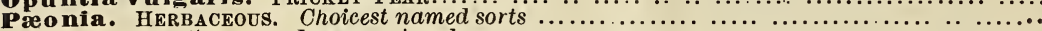
In separate colors. Mixed

Pardanthus Sinensis. CHINESE BLACKBERRY LILY

(n)

Phlox amoema. DWARF OR LOVELY PHLOX.

PERENNIAL or GARDEN. A large collection of named sort.

PERENNIAL Or GARDEN. A targe collection of named sort

Carolina ovata. LAUREL-Leaved PHLO

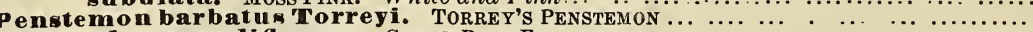

Platycodongiandiflorum. GREAT BeLL FLOWER

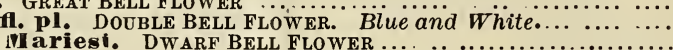

Plumbago Larpenta. LEADWORT.

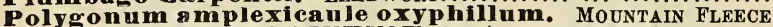

SACHA I INENSE (true). SACALINE. 1 yr

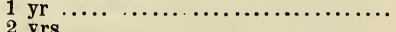

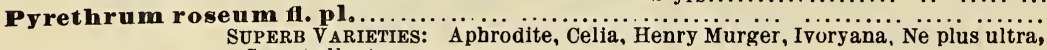
SUPERB VARIETI

Tsehihatchewii. TUFTING DaISY

nliginosum. GIANT DAISY.

Hanunculus acris fi. pl. Bachelor's BUTTON or DOUBLE BUTTERCUP.

Tanme maxima. GReAT CONE FLOWER

Fudbeckia max

Salvia lyrata....

"s sectabile. SHOWY STONECROP.

spiraea lobata (venusta). QUEeN OF THE PRAIRIE. filifera fl. D. DOUBLE SNEkZEWORT.

palmata plegans

tachys lamata. WOOLLY WOUNDWORT

Statice latifolia. SEA LAVENDER, strong piants.

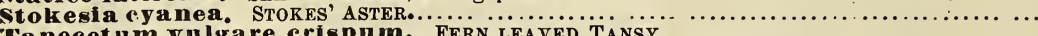

Tanecetum vulgare crispum. FERN LEAVED TANSY

Thymus montanus albus. WBITE MOUNTAIN THYME.

Tradescantia virginica. WIDOw's TEARs.

Tunica saxifraga. ROck TUNICA

$$
\text { coccinea... }
$$

Valeriama oficinalis. WILD HELIOTROPE.

veronica amathystina. AMETHYST SPEEDWELL.

$$
\begin{aligned}
& \text { incana } \ldots \text {............. } \\
& \text { repens. THYME LEAVED VERONICA } \\
& \text { rupestris } \\
& \text { spicata. }
\end{aligned}
$$

viola odorata "cailiforiä̈;

pedata. 66 Double Fussian;

HARDY GRASSES.

Eulalia gracillima univittaia \begin{tabular}{c} 
Japonica \\
\hdashline
\end{tabular}

clumps

Uniola latifolia.

\section{BULBS, ROOTS AND TUBERS.}

\section{CALLA LILIES.}

Doz. 100

CALLA ETH I, PICA. 13/4 to 2 in. diam.... \$1 $00 \quad \$ 650$

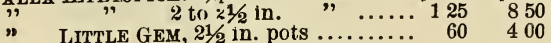

\section{CANNAS.}

Alphonse Bouvier.

Chas. Henderson

CHEVELIFR

EGANDALE.

FLORFNCE VAÜGHAN

FRANCOIS CROZY.

HENRY A. DREER.

J. D. CABOS.

in ad. Crozy.

PAUL BRUANT.......

PAUL MARQUANT.................... 50

800
1000

Queen rliarlotte.

$\begin{array}{rr}\text { Doz. } & 100 \\ \$ 125 & \$ 800\end{array}$ $\$ 800$
300

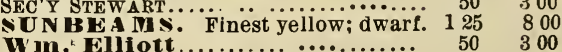

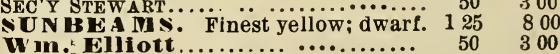

\section{DAHLIAS.}

$250 \quad 2000$

$300 \quad 2000$

$300 \quad 2000$

$\begin{array}{lll}400 & 3500 \\ 300 & 2500\end{array}$

$300 \quad 2500$

$\begin{array}{ll}300 & 2000 \\ 300 & 2500\end{array}$

$\begin{array}{ll}300 & 2500 \\ 300 & 2500\end{array}$

$250 \quad 2000$

$300 \quad 2500$

CACTUS, NYMPHCA and other fine named varieties ....................... $150 \quad 1000$ LARGE.FLOWERED and POMPONE, named varieties ....

Hy ACINTHs, Double and Single, flne sorts

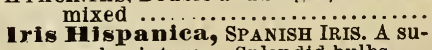
Tulips. Mixed. A fine mixture of best pouble and Single varieties ......
5000

5000

7000

1000

$\$ 2500$

2500 perb mixture. Splendid bulbs. . 


\section{ROSES.}

FIELD-GROWN ROSES.

Doz.

100

Climbing, 2 and 3 years.

ANNE MARIA (Pride of Washington), BALTIMORE BELLE, GEM OF THE PRAIRIE, GREVILLE

$\$ 100 \$ \$ 600$ T SEVEN SISTERS. PRAIRIE QUERN, RUSSELI'S COTTAGE.

Clothilde soupert. 1 gr. fine plants.

$150 \quad 1000$

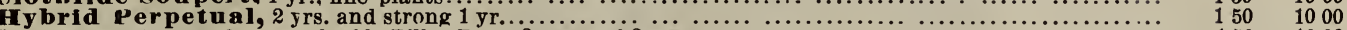

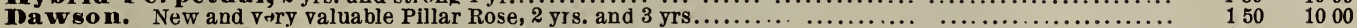

Wichuriana. Japan Trailing, strong plants.

Rosa rugosa. See Deciduous Shrubs, page 10.

Bride $21 / 2$ in. pots

BON SILENE........................2\% in. "

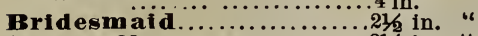

CAROLINE MARINESSE..............21: in. "

CLIMBING METEOR................21 in. "

Clothilde So upert............21: in. ":

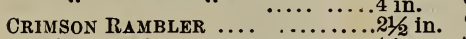

DUCHESSE DE BRABANT. ...........216 in. "

Empress of china .............21/2 in. "

GEORGE PERNET................... "

GOLDEN FAIRY. ...............

GOLDEN GATE. ....................

Gol." G.. ................

Kaiserine ing. vietoria..21/. in.

LA FRANCE ................

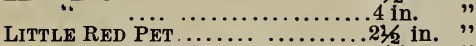

Mad. CaROLINE TESTOUT.............21/2 in. ",
Doz. 100

$150 \$ 350$

$\begin{array}{rr}100 \\ 50 & 350\end{array}$

$100 \quad 600$

$50 \quad 350$

50 350

150

$100 \quad 600$

$100 \quad 600$

$150 \quad 1000$

$150 \quad 350$

$\begin{array}{lll}75 & 5 & 00\end{array}$

150
50 300

50

100

$30 \quad 350$

150
60 400

$\begin{array}{rr}60 & 400 \\ 125 & 800\end{array}$

$60 \quad 400$

$125 \quad 800$

$50 \quad 350$

$\begin{array}{lll}100 & 1000\end{array}$
MAD. PERnET DUÇ, "... ......21/2 in. pots Maman Cochet.............. 4 in. in. " Meteor.... . . . .................... in. nignonette.

MARECHAL NIEL

MARIE LAMBERT................

Mrs. Degraw ................

......4 in.

$"$ " PIER " MONGA..........21 in.

PAPA GONTIER .....................21/2 in.

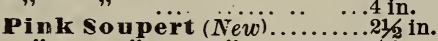

$"$ "........4 in. "

QUEEN'S SCARLET..................21/2 in. "

sanguinea .....................21/6 in. "

TRIOM PHE dE PERNET PERE..........

WichURIANA $\ldots \ldots \ldots \ldots \ldots \ldots .21 \%$ in. " $\begin{array}{rr}150 & 1000 \\ 125 & 800\end{array}$

Doz. $\quad 100$

$\$ 60 \quad \$ 400$

$150 \quad 1000$

$\begin{array}{lll}50 & 350\end{array}$

$60 \quad 400$

$150 \quad 1000$

$\begin{array}{rr}50 & 300 \\ 00 & 600\end{array}$

150

$\begin{array}{rr}50 & 350 \\ 50 & 350\end{array}$

$\begin{array}{rr}50 & 350 \\ 100 & 600\end{array}$

100

$150 \quad 1000$

$60 \quad 400$

$150 \quad 1000$

150

50300

$100 \quad 600$

50
300

60
600

$150 \quad 1000$

50
300

\section{PLANTS IN POTS.}

Abutilon, SOd. DE BONN, ECLIPSE, GOLDEN BELLS.............................21/2 in. Ageratum. том тномв (Little Gem). Liт-

A TLE DORRITT . . . . . . . . . . 21/2 in. mous flowers, beautifully striped and

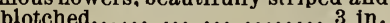

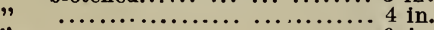

Araucaria imbricata (Monkey Puzzle),

$4 \mathrm{in.} \mathrm{pots,} 10$ to $15 \mathrm{in}$. high...... $21 \% \ldots \ldots$.

Begonias, FLOW ERING VARIETIES, 21/2-3 in.

Coleus. ten most popular sorts. ........21

Cuphæa Llavæ.. ............ $2 \frac{1}{2}$ in

Echeverias, SECUNDA, SECUNDA GLAUCA, SECUNDA GLAUCA MAJOR, good plants...

Fuchsias, twenty superb varieties, . $21 / 3$ in

GREVILU EA ROBU SA (Silk Oak)

Too much can hardly be said in praise of this wond plant. It is 5 . nerb for decorative purposes, growing wine rapidly than any Palm and easily cart : for. The foliage is long and drooping, finely and deeply cut like a Fern, the young leaves being of a like a Fern, the young leaves being of a light bronzy shade and line tips covered with a soft down resembling raw silk. Its unexce!led for house culture. Superb well-furnished plants, $31 / 2$ in. pots, 10 to

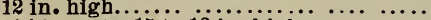
$31 / 2$ in. pots, 15 to 18 in. high ................

GER A $\mathbf{I}$ IIM, GEN. GRANT, fline scarlet,

" $\quad$. G. HILL, flne salmon pink; semidouble ....................... BEAUTE POINTEVIN, superb salmon; semi-double ............21/2 in
AFAVORITE, flnest white; double.

" LAFAVORITE, flnest white; double.

" ROSEBUD...........................

$" \quad$ ROSEBUD ........ $\ldots$ iz finest varieties. Gelsemium sempervirens ( Yellow Jessamine). $21 / 2$ in.

German Ivy....................

Gynura aurantia ca(Velvet Plant) $21 / 6$ in

IVy, ENGLISH Or IRISH................

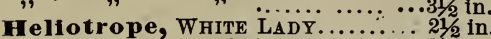

Heliotrope, WHITE LADY..........21/2 in.

Laris Daisy or Marguerites (Chrusanthemum frutescens)...........21/2 in.

Petunia, Double .................21: in.

Smilax, strong plants.............21/2 in.

Toyal Purple).... ........216 in

Tecoma Smithii $($ Reyal Purple,.........21/2 in.

\section{$\$ 60$}

40

300

$400-3000$

750

50

60
40

60

75

125
150

175

50

60

60

50

60
50

75

125
40

150

1 on

1 .

60

60

75

100

40

50

125
2000

$\$ 4 \mathrm{CO}$

250

4000

350
400

250

400

500

350

800

1000

350

400

400

350

350

500

800
250

600

400

400

500

600

350

300

800

800
1200

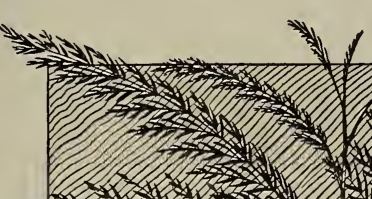

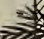
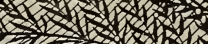
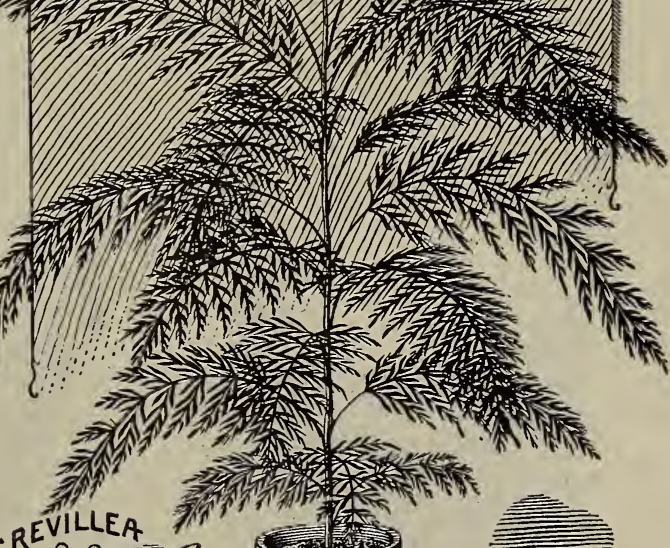
ROB
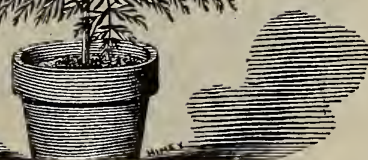

Thrysacanthus rutilans (Carmine Fountain)....................21/3 in. $\$ 100$

Tradescantias, three sorts........21/2 in.

" " Periwinkle). 21/5 in. $100 \quad 600$

VIOLET, CALIFORNIA ............21/2 in. $\quad \begin{array}{rr}150 & 1000 \\ 350\end{array}$

Washingtonia filifera, superb plants, " robusta, su. $150 \quad 1000$

perb plants....................2 21/2 in. $150 \quad 1000$ A2UATIC PLANTS.

Parrot's Featlier (Myriophyllum Proserpinacoides) ......................

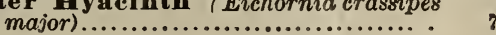




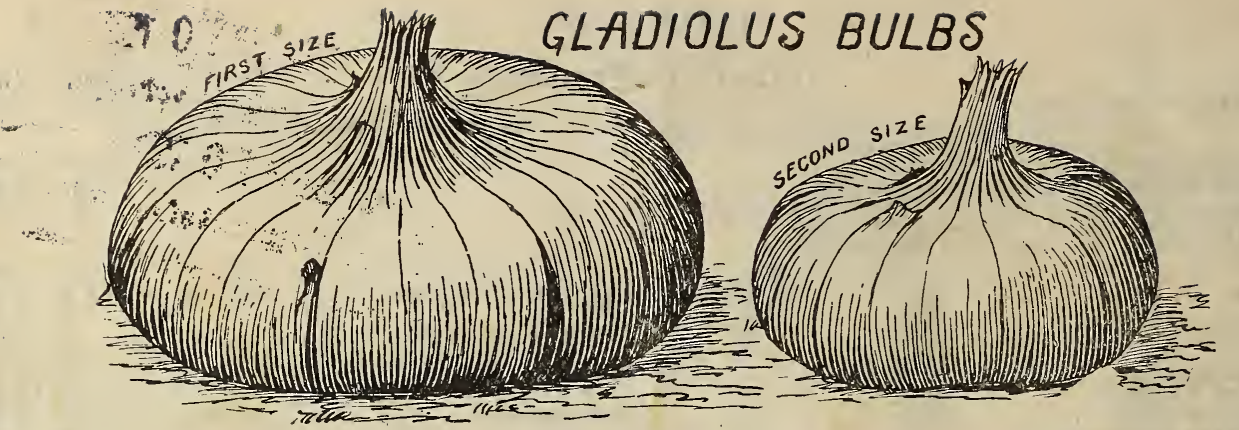

Experience has proved that the climate and soil of Monmouth, here by the seaside, with our long season of growth and bright sunshine and the heat of summer tempered by the ocean, is the most fav-

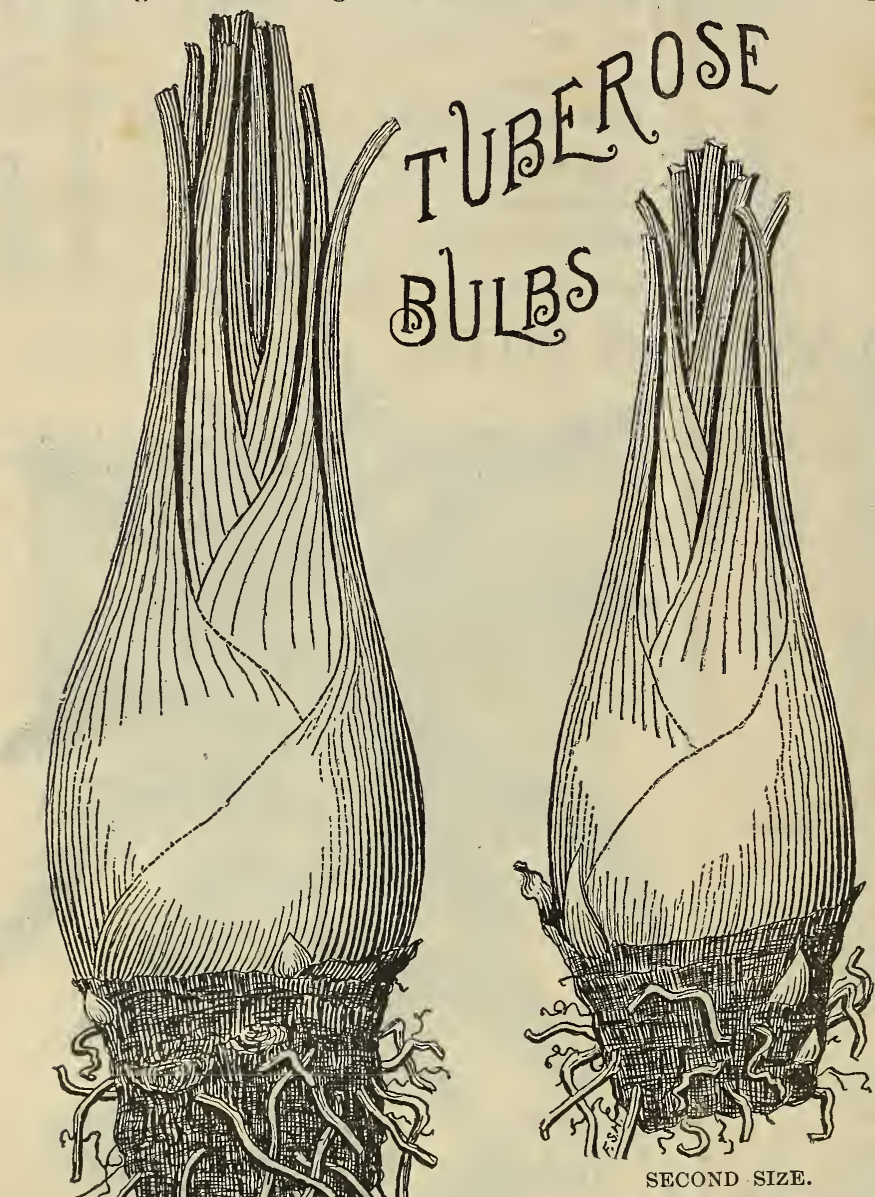
orable spot upon the globe for the perfect development of the Gladiolus. Our bulbs are therefore not only equal, but are superior to those produced anywhere else in America or Europe.

ALL COLORS MIXED; CHIEFLY RED.

First size, 100, 75c; 1000, $\$ 5.00$. Second size, 100, 50c; 1000, $\$ 3.50$.

Monmouth Strain.

Composed entirely of superb named varieties; sixty per cent white and light colored; not over twenty per cent red; balance yellow and variegated. The finest strain of Gladiolus in commerce. Particularly valuably for florist's use. First size, (See cut), 100, $\$ 1.25,1000, \$ 6.00$.

Second size, (See cut), 100, $\$ 1.00$ $1000, \$ 4.50$.

Mad. Lemoine, 1st size, 100, $75 \mathrm{c} ; 1000, \$ 5.00$.

Second size, $100,50 c ; 1300, \$ 3.50$.

\section{TUBEF USE BULBS.}

Southern grown Tuberose bulbs of superior quality. They are exceedingly bright and handsome and in perfect condition, strictly true to name and pure. The average sizes are accurately shown in the accompanying illustration.

Excelsior Pearl, Ist size, (4to 6 in.), 100, \$1.00; 1000, \$8.00. 2d size 3 to 4 1-2 in.), $100,85 \mathrm{c} ; 1000, \$ 5.00$.

CALADIUM ESCULENTUM.

EXCELSIOR PEARL

1 st -ize, 10 to 12 in. circumference, doz., $\$ 1.00 ; 100, \$ 7.00$.

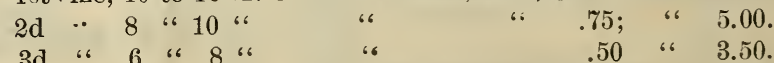

\section{OXALIS BULBS.}

Our bulns of Oxalıs are very fine and are much larger than thcse usually sent out, as is show $\mathrm{n}$ by the illustration.

Dieppi. 100, 20c; 1000, \$1.25.

Lasiandra........ "6 25e; 66

1.50 .

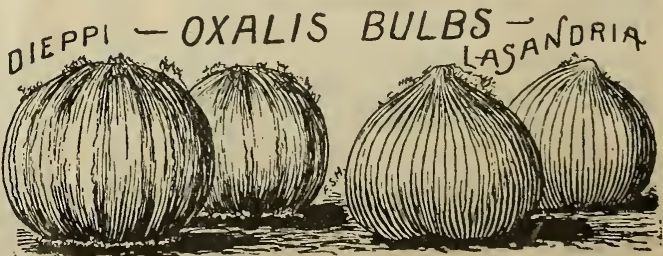

